\title{
Les facteurs influençant le rendement oléicole : cas de la région de Jijel du Nord-Est algérien
}

\author{
Moussa Lachibi ${ }^{1, *}$, Fouèd Chehat ${ }^{2}$ et Fathi Abdellatif Belhouadjeb ${ }^{2}$ \\ ${ }^{1}$ L’École Nationale Supérieure d'Agronomie, El Harrech, (ENSA Algérie), El Harrech, Algeria \\ ${ }^{2}$ L'Institut National de la Recherche Agronomique d'Algérie, INRAA, El Harrach, Algeria
}

Reçu le 9 octobre 2018 - Accepté le 4 mars 2019

\begin{abstract}
Résumé - La région de Jijel, dans le Nord-Est algérien, possède un important potentiel naturel oléicole favorisant une activité économique importante autour de ce produit, notamment dans le milieu rural. Néanmoins, la production des exploitations de la région demeure faible et instable dans le temps, malgré les efforts de plantations entrepris depuis 2001. La recherche des causes de cette faiblesse nous a conduits à repérer les principaux facteurs spécifiques ayant un impact significatif sur les rendements. À cet effet, quatre facteurs ont été identifiés comme ayant des effets significatifs sur les rendements des exploitations oléicoles locales : deux s'avèrent liés à la variété et les deux autres à l'environnement. Les facteurs intrinsèques sont l'origine de la variété (locales et hors région) et l'âge des plants. Les deux autres facteurs extrinsèques (liés à l'environnement) sont l'altitude et la pente des terrains; les exploitations se situant à des altitudes supérieures à 600 mètres, avec des pentes modérées enregistraient des rendements plus importants. Cette étude nous a permis de connaître les exigences agro-écologiques et les principaux facteurs spécifiques à prendre en considération pour une bonne affectation de la ressource oléicole au niveau local.
\end{abstract}

Mots clés : agriculture / territoire / facteurs / variété / rendement / effet

\begin{abstract}
Factors influencing olive oil yield: case of the Jijel region of northeastern Algeria. The Jijel region, in northeastern of Algeria, has a significant natural olive-growing potential, which favors significant economic activity around this product, particularly in rural areas. Nevertheless, the production of farms in the region remains weak and unstable over time, despite the planting efforts undertaken since 2001. The search for the causes of this weakness led us to identify the main specific factors having a significant impact on yields. To this end, four factors have been identified as having significant effects on the yields of local olive farms; intrinsic factors are the origin of the variety (local and out of region) and the age of the plants. The other two extrinsic factors (related to the environment) are the altitude and slope of the land; farms at altitudes above 600 meters with moderate slopes recorded higher yields. This study allowed us to know the agro-ecological requirements and the main specific factors to consider for a good allocation of the olive resource at the local level.
\end{abstract}

Keywords: agriculture / territory / factors / variety / yield / effect

\section{Introduction}

L'économie oléicole mondiale vit une globalisation de la demande. L'offre de son côté évolue sur base de nouveaux modèles de production agricole (Barjol, 2014). La filière oléicole algérienne montre, depuis des décennies, des signes de crise, avec une dualité entre un système traditionnel peu compétitif en raison des conditions géomorphologiques (dans les zones de montagne) et institutionnelles (faible soutien) et

\footnotetext{
*Correspondance : moslachibi18@yahoo.fr
}

un système moderne, destiné principalement à la production de l'olive de table (Hadjou et al., 2013).

La région de Jijel, de par son positionnement géographique est considérée parmi les régions du Nord-Est de l'Algérie les plus productives en olives, avec $6 \%$ de la production nationale. Elle est aussi un pôle de transformation industrielle des olives, ce qui a constitué une source importante de revenu pour les acteurs de la région.

Cependant, la production oléicole de la région est constatée comme faible et ne progresse pas malgré les efforts consentis pour son augmentation, particulièrement à partir de l'année 2000, début du programme national oléicole. Dont l'un de ses 
Tableau 1. Connaissance des variétés cultivées.

\begin{tabular}{llc}
\hline Connaissance de la variété & Nb.cit & Fréquence \\
\hline Oui & 57 & 90,5 \\
Non & 6 & 9,5 \\
Total & 63 & 100 \\
\hline
\end{tabular}

Source: Calculé à partir des données de l'enquête.

objectifs la création de nouvelles plantations à différentes densités en fonction des conditions agro-climatiques (Mendil, 2009). L'analyse des données de la production annuelles de la région allant de 2001-2015 (DSA, 2016), montre que la production annuelle, le long de la période, n'a pas dépassé le niveau de la production de 2001, exception faite pour l'année 2009 avec une différence de 9638 quintaux.

Cette situation inquiétante de la filière oléicole locale nous amène à nous poser la question de recherche suivante: Quels sont les facteurs influençant les rendements oléicoles dans le Nord-Est algérien?

\section{Méthodologie}

La région de Jijel possède un potentiel oléicole important qui représente $6 \%$ du potentiel national, l'oléiculture occupe une place importante dans l'assiette foncière agricole de la région, elle représente environ $34 \%$ de la superficie agricole utile (SAU) et $80 \%$ du potentiel arboricole (DSA, 2016).

Nous avons procédé à la réalisation d'une enquête sur terrain auprès des oléiculteurs de la région à l'aide d'un questionnaire. Cet échantillon est obtenu à partir d'une sélection raisonnée prenant les exploitations ayant des superficies supérieures ou égales à 3 hectares, ce qui nous a permis d'avoir 63 exploitations représentatives. En effet, le choix de la superficie de 3 hectares se renvoi à des objectifs à savoirs :

- une taille de référence pour une masse informationnelle importante;

- une bonne identification des pratiques culturales et des systèmes de cultures ;

- accès facile à l'exploitation.

Concernant le choix des facteurs influençant les rendements, il est basé sur les réponses des oléiculteurs et aussi sur les spécificités de la région, notamment des spécificités agroécologique pour des vergers oléicoles répartis dans toute la chaîne montagneuse de la région et dans les collines sur des sols argilo-rocheux aux caractéristiques hydrographiques denses et abondantes, et climatique avec des précipitations soutenues sur tout le territoire de la wilaya, la pluviométrie moyenne étant de $1200 \mathrm{~mm} / \mathrm{an}$. Et, des spécificités au territoire avec, notamment la présence de plusieurs variétés, les facteurs choisis sont: la variété (de la région et hors de la région), l'âge des plants, l'altitude et la pente.

\section{Résultats et discussions}

Compte tenu de la réalité locale et des caractéristiques des systèmes de production de la région, et afin de mesurer l'impact des facteurs qualitatifs sur les rendements en olive, nous procédons à une analyse statistique mettant en relief leurs effets.

Pour cela, nous nous intéresserons aux principales variables qualitatives qui ont des effets apparents sur les rendements, sur la base des appréciations des oléiculteurs, ce qui suppose qu'il n'y a pas une mesure fiable du degré de contribution de chaque variable à l'amélioration de rendement dans les stades agro-écologiques caractérisant la région.

Les principales variables considérées (contrôle) pour analyser cette réalité sont les suivantes: l'origine de la variété; l'âge des arbres; l'altitude et la pente.

\subsection{La variété}

Parmi les facteurs qui conditionnent la productivité, on citera le facteur variétal et les conditions pédoclimatiques. Pour cela, l'emploi de variétés plus adaptées dans les zones à vocation oléicole est l'une des premières conditions pour obtenir de plus grandes productions (COI, 2007). La connaissance de la variété par l'oléiculteur se matérialise à travers la vérification des éléments suivants :

- lors de l'achat des plants, généralement, le nom de la variété est la première information demandée par l'acheteur et fournie par le vendeur;

- la présence d'un certificat de qualité se présentant sous la forme d'une étiquette placée sur le plant, mentionnant le nom de la variété et de la pépinière de production;

- l'origine de la variété notamment dans le cas des plants transférés à partir d'une autre exploitation (Tab. 1).

Les enquêtés ont donc été interrogés sur leurs connaissances, des caractéristiques et des variétés qu'ils ont plantés, et plus de $90 \%$ d'entre eux ont affirmé les connaître. Les oléiculteurs interrogés pouvaient préciser l'origine des variétés cultivées (Tab. 2).

L'analyse des données montre que 49,20\% des exploitations enquêtées cultivaient uniquement la variété de la région et $34,92 \%$ des exploitations cultivaient uniquement des variétés hors région, par contre $15,87 \%$ cultivaient des variétés mixtes.

Les variétés dites de la région: les principales par ordre d'importance sont:

- Al Hamra : éparpillée dans la majorité des exploitations de la région, considérée comme la plus productive chez plus de $70 \%$ des oléiculteurs, résistante aux maladies, avec en plus l'aspect vigoureux de l'arbre, elle s'adapte à tous les étages agro-écologiques de la région. Un seul inconvénient a été évoqué; celui de sa taille volumineuse engendrant des difficultés lors des travaux d'entretien et lors de la récolte;

- Al Kahla: de même capacité productive qu'Al Hamra, sa présence dans l'assiette oléicole de la région est faible par rapport à la précédente. Elle est surtout rencontrée dans les communes d'El Amir et de Texana;

- la Rougette d'El Amir: elle s'adapte mieux aux étages agro-écologiques inférieurs à 500 mètres d'altitude; elle est moins productive que les deux précédentes mais résistante aux maladies. 
Tableau 2. Connaissance de l'origine de la variété.

\begin{tabular}{lcccc}
\hline \multicolumn{4}{c}{ Frequency table : origine de la variété (Feuil 1 in données 2) } \\
\hline & Nombre & $\begin{array}{l}\text { Nombre- } \\
\text { cumulatif }\end{array}$ & Pourcentage & $\begin{array}{l}\text { Pourcentage- } \\
\text { cumulatif }\end{array}$ \\
\hline Hors région & 22 & 22 & 34,92063 & 34,9206 \\
De la région & 31 & 53 & 49,20635 & 84,1270 \\
$\begin{array}{l}\text { De la région et } \\
\text { hors région }\end{array}$ & 10 & 63 & 15,87302 & 100,0000 \\
Missing & 0 & 63 & 0,00000 & 100,0000 \\
\hline
\end{tabular}

Source : Calculé à partir des données de l'enquête.

Les variétés Bouchouka et Chouagri sont également locales et obtiennent des niveaux de production comparables avec les autres variétés, mais elles sont faiblement présentes dans la région par rapport aux deux précédentes.

Les variétés dites hors région: ce sont des variétés qui n'ont pas un ancrage local, mais qui ont été introduites récemment par le marché ou par le dispositif de soutien. Celles qui sont cultivées actuellement sont:

- la Chemlal: sa présence dans le verger oléicole local ne cesse d'augmenter et elle occupe aujourd'hui une part importante des superficies la plaçant en deuxième position après Al Hamra, notamment parce que l'essentiel des soutiens accordés à l'oléiculture a reposé sur cette variété ;

- la Chemlal tunisienne: variété d'origine tunisienne, de bonne qualité sur le plan productivité en olives ainsi que sur le plan adaptation et facilité des travaux d'entretien et de récolte. Méconnue au niveau de la région et présente chez un nombre limité d'oléiculteurs, elle est peu demandée. En Tunisie cette variété participe à plus de $80 \%$ dans la production nationale d'huile d'olive (Gharbi et al., 2014);

- Azeradj : elle ressemble, sur le plan morphologique, aux variétés locales, mais elle est moins productive et présente des difficultés lors de la récolte. Par contre, dans la région de Béjaia et d'après (Lamani et Ilbert, 2016), cette variété a l'avantage d'être la seule à assurer un rendement en huile allant de 24 à $28 \%$, qui est meilleur que les autres variétés pour lequel ce rendement est d'environ 16 à $24 \%$. Le second atout de cette variété est sa résistance à la sècheresse.

Les principales appréciations des oléiculteurs sur la qualité des différentes variétés oléicoles cultivées dans la région, sont basées notamment sur deux critères : le niveau de productivité et les performances apparentes de chacune d'entre elles. La connaissance de la variété oléicole par les exploitants enquêtés a permis de préciser la nature des différentes variétés présentes dans les exploitations (Tab. 3).

Il ressort que les variétés oléicoles Al Hamra et Chemlal sont les plus reconnues et les plus présentes dans les exploitations oléicoles locales, avec un taux de présence égale à 45,71\% pour la variété Al Hamra, parce qu'elle est considérée comme originaire de la région, et en deuxième position, il y a la Chemlal avec un taux de $25,71 \%$, cette variété est la plus disponible sur le marché, notamment
Tableau 3. Variétés cultivées au niveau des exploitations.

\begin{tabular}{lll}
\hline Variétés & Nb.cit & Fréquence (\%) \\
\hline Al Hamra & 32 & 45,71 \\
Chemlal & 18 & 25,71 \\
Rougette d'El Emir & 5 & 7,14 \\
Al Kahla & 4 & 5,71 \\
Sigoise & 3 & 4,28 \\
Azeraj & 2 & 2,85 \\
Espagnole & 2 & 2,85 \\
Bouchouka & 1 & 1,42 \\
Chamlal Tunisienne & 1 & 1,42 \\
Chouagri & 1 & 1,42 \\
Jarazia & 1 & 1,42 \\
Total & 70 & 100 \\
\hline
\end{tabular}

Source: Calculé à partir des données de l'enquête.

depuis le démarrage des soutiens à l'oléiculture grâce auxquels elle était presque la seule variété livrée par le programme.

La Rougette d'El Emir et Al Kahla sont des variétés issues de la région et représentent respectivement 7,14 et 5,71\% des déclarations; elles sont généralement plantées dans les anciennes exploitations ou héritées de la période coloniale.

La Sigoise est une variété originaire de l'Ouest, elle est nouvellement introduite par le dispositif de soutien pour diversifier la production, notamment dans les zones de montagne. D'autres variétés sont aussi présentes mais la place qu'elles occupent est très faible, on peut citer les variétés Bouchouka, Chouagri et Jarazia sont considérées comme locales et implantées uniquement dans des zones bien identifiées, par contre, Azeradj et la Chemlal tunisienne sont des variétés externes à la région (Tab. 3).

L'analyse des données (Tab. 4) montre que les exploitations qui ont pu mélanger les variétés locales avec des variétés hors région (mixte) ont enregistré des rendements moyens supérieurs à la moyenne de la wilaya qui est de 20 litres/ quintal. Ainsi, l'effet des variétés locales est très significatif puisque le groupe concerné rejoint celui des variétés mixtes (région et hors région). Par contre, l'effet des variétés hors région est faible et le rendement moyen de ce groupe est inférieur à la moyenne.

\subsection{L'âge des plants}

Les rendements des oliviers en olive sont variables en fonction de l'âge des arbres, les données du tableau (Tab. 5) relatives aux âges des oliveraies montrent que la classe d'âge moyen est la plus présente dans les exploitations avec $47,61 \%$, par contre la classe des plants âgés en deuxième position présente une part considérable avec $39,68 \%$, et la classe d'âge jeune représente seulement $12,69 \%$. L'analyse de l'effet de l'âge des plants sur les rendements est illustrée dans le tableau 6.

Les résultats présentés nous montrent que l'âge a un effet significatif sur les rendements. Ainsi, le groupe des exploitations ayant des oliveraies d'âge moyen enregistre un 
Tableau 4. Analyse de l'effet de l'origine de la variété sur le rendement.

LSD test; variable rendement (Feuil 1 in données3) Homogenous Groups, alpha $=0,05000$ Error : Between $\mathrm{MS}=11,270, \mathrm{df}=60,000$

\begin{tabular}{|c|c|c|c|c|}
\hline & $\begin{array}{l}\text { Origine de } \\
\text { la variété }\end{array}$ & $\begin{array}{l}\text { Rendement- } \\
\text { Mean }\end{array}$ & 1 & 2 \\
\hline 3 & Hors région & 18,63636 & & $* * * *$ \\
\hline 1 & De la région & 20,83871 & $* * * *$ & \\
\hline 2 & De la région et hors région (mixte) & 21,90000 & $* * * *$ & \\
\hline
\end{tabular}

Source: Calculé à partir des données de l'enquête.

Tableau 5. L'âge des oliveraies des exploitations.

\begin{tabular}{llccc}
\hline \multicolumn{4}{c}{ Frequency table : Age arb (Feuil 1 in données 2) } \\
\hline & Nombre & $\begin{array}{l}\text { Nombre- } \\
\text { cumulatif }\end{array}$ & Pourcentage & $\begin{array}{l}\text { Pourcentage- } \\
\text { cumulatif }\end{array}$ \\
\hline Âge J & 8 & 8 & 12,69841 & 12,6984 \\
Âge A & 25 & 33 & 39,68254 & 52,3810 \\
Âge M & 30 & 63 & 47,61905 & 100,0000 \\
Missing & 0 & 63 & 0,00000 & 100,0000 \\
\hline
\end{tabular}

Source : Calculé à partir des données de l'enquête. Âge jeune $(\mathrm{J}): 0$ 15 ans. Âge moyen (M) : 16-50 ans. Âgé (A) : $>50$ ans.

rendement moyen supérieur à la moyenne, soit 22,36 litres/ quintal, le douzième groupe des exploitations ayant des oliveraies d'âge avancé enregistre un rendement moyen inférieur à la moyenne, par contre le troisième groupe des exploitations ayant de jeunes plantations enregistre un faible rendement moyen (16,12 litres/quintal).

\subsection{L'altitude}

Le stade agro-écologique sur lequel se cultive les plants requiert une importance cruciale dans leurs adaptations et affecte leurs niveaux de production. De ce fait, l'altitude constitue un facteur important mesurant l'intervalle adéquat pour une bonne adaptation écologique de la variété.

Nous remarquons d'après les données (Tab. 7) que 58,73\% des exploitations enquêtées se situent à des altitudes inférieures à 600 mètres, par contre les autres $(41,27 \%)$ se situent à des altitudes supérieures à 600 mètres. Cependant le niveau d'altitude de 600 mètres est pris comme référence puisqu'il est considéré comme point critique pour l'adaptation de la variété Chemlal.

L'analyse de l'effet de l'altitude (Tab. 8), nous fait ressortir deux groupes: le premier est constitué des exploitations qui sont situées dans un intervalle d'altitude inférieur à 600 mètres, par contre, le deuxième groupe des exploitations qui se localisent à une altitude supérieure à 600 mètres enregistrent un rendement moyen supérieur à la moyenne (22,71 litres/quintal).
Tableau 6. Analyse de l'effet de l'âge des oliviers sur le rendement. LSD test; variable rendement (Feuil 1 in données 3) Homogenous groups, alpha $=0,050$

\begin{tabular}{llllll}
\hline & Âge arb & Rendement-Mean & 1 & 2 & 3 \\
\hline 3 & Âge J & 16,12500 & $* * * *$ & & \\
1 & Âge A & 19,00000 & & $* * * *$ & \\
2 & Âge M & 22,36667 & & & $* * * *$ \\
\hline
\end{tabular}

Source : Calculé à partir des données de l'enquête.

Tableau 7. Position des exploitations en altitude.

\begin{tabular}{|c|c|c|c|c|}
\hline \multicolumn{5}{|c|}{ Frequency table: altitude (Feuil 1 in données 2 ) } \\
\hline & Nombre & $\begin{array}{l}\text { Nombre- } \\
\text { cumulatif }\end{array}$ & Pourcentage & $\begin{array}{l}\text { Pourcentage- } \\
\text { cumulatif }\end{array}$ \\
\hline 0 & 5 & 5 & 7,93651 & 7,9365 \\
\hline 10 & 1 & 6 & 1,58730 & 9,5238 \\
\hline 20 & 1 & 7 & 1,58730 & 11,1111 \\
\hline 50 & 6 & 13 & 9,52381 & 20,6349 \\
\hline 60 & 1 & 14 & 1,58730 & 22,2222 \\
\hline 100 & 5 & 19 & 7,93651 & 30,1587 \\
\hline 300 & 4 & 23 & 6,34921 & 36,5079 \\
\hline 400 & 8 & 31 & 12,69841 & 49,2063 \\
\hline 500 & 6 & 37 & 9,52381 & 58,7302 \\
\hline 600 & 12 & 49 & 19,04762 & 77,7778 \\
\hline 700 & 5 & 54 & 7,93651 & 85,7143 \\
\hline 800 & 7 & 61 & 11,11111 & 96,8254 \\
\hline 900 & 2 & 63 & 3,17460 & 100,0000 \\
\hline Missing & 0 & 63 & 0,00 & 100,0000 \\
\hline
\end{tabular}

Source: Calculé à partir des données de l'enquête.

Tableau 8. Analyse de l'effet de l'altitude sur le rendement.

LSD test; variable rendement (Feuil 1 in données 3) Homogenous groups, alpha $=0,05$

\begin{tabular}{lllll}
\hline & Altitude & $\begin{array}{l}\text { Rendement }- \\
\text { Mean }\end{array}$ & 1 & 2 \\
\hline 2 & B & 19,16667 & $* * * *$ & \\
1 & A & 20,10526 & $* * * *$ & \\
3 & C & 22,71429 & & $* * * *$ \\
\hline
\end{tabular}

Source : Calculé à partir des données de l'enquête.

\subsection{La pente}

L'hétérogénéité du relief de la région ainsi que les exigences agro-écologiques de chaque variété oléicole cultivée nous incitent à classer les exploitations par rapport à la pente et leurs effets sur les rendements.

L'analyse des données (Tab. 9) nous montre que la plupart des exploitations enquêtées sont dans des positions de pente, ce qui explique la nature montagneuse des reliefs, soit $69,84 \%$. 
Tableau 9. Classification des exploitations par rapport à la pente.

\begin{tabular}{llccc}
\hline \multicolumn{5}{c}{ Frequency table: pente (Feuil 1 in données 2) } \\
\hline Nombre & $\begin{array}{l}\text { Nombre- } \\
\text { cumulatif }\end{array}$ & Pourcentage & $\begin{array}{l}\text { Pourcentage- } \\
\text { cumulatif }\end{array}$ \\
\hline Pente & 44 & 44 & 69,84127 & 69,8413 \\
Plat & 13 & 57 & 20,63492 & 90,4762 \\
Plat-pente & 6 & 63 & 9,52381 & 100,00 \\
Missing & 0 & 63 & 0,00000 & 100,00 \\
\hline
\end{tabular}

Source : Calculé à partir des données de l'enquête.
Tableau 10. Analyse de l'effet de la pente sur le rendement.

LSD test; variable rendement (Feuil 1 in données 3) Homogenous groups, alpha $=0,50$

\begin{tabular}{lllll}
\hline & Pente & $\begin{array}{l}\text { Rendement }- \\
\text { Mean }\end{array}$ & 1 & 2 \\
\hline 2 & Pente & 19,45455 & $* * * *$ & \\
1 & Plat & 20,92308 & $* * * *$ & \\
3 & Plat-pente & 24,50000 & & $* * * *$ \\
\hline
\end{tabular}

Source : Calculé à partir des données de l'enquête.

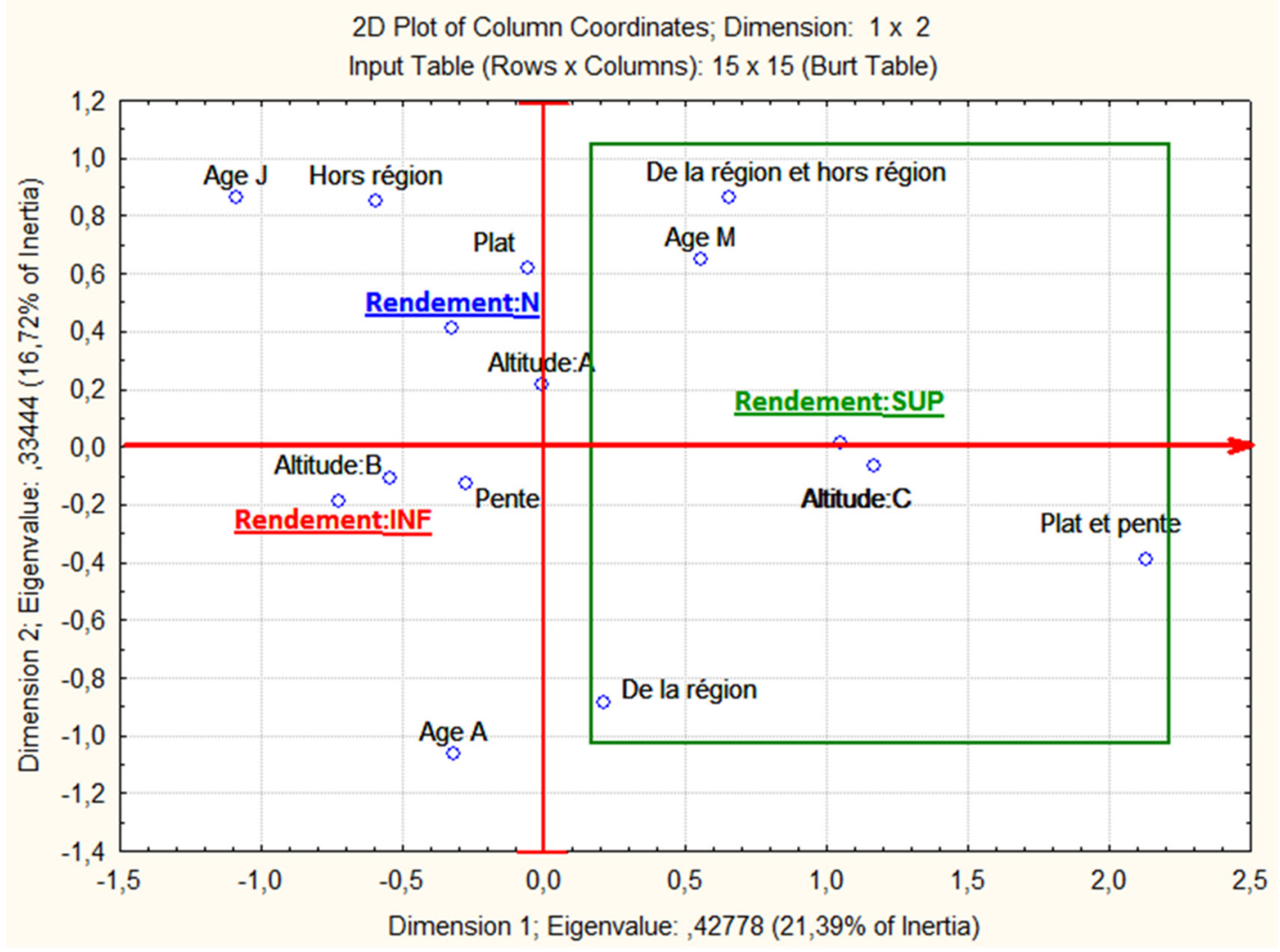

Fig. 1. Analyse des correspondances multiples.

Les exploitations situées dans des zones plateaux représentent $20,63 \%$ de l'ensemble, par contre les pentes modérées représentent seulement $9,52 \%$.

L'analyse des données (Tab. 10) fait ressortir que la variable pente a un effet significatif sur les rendements des exploitations. On distingue deux groupes de rendements relatifs à cette variable: le premier groupe a un rendement moyen égal à 20,92 litres/quintal et 19,45 litres/quintal, respectivement pour les exploitations qui se situent aux terrains plats ou à pente faible ; le deuxième groupe des exploitations situées sur des terrains à pentes modérées ou à niveau mixte (pentes modérées et fortes) enregistrent un effet très significatif, le rendement moyen étant de 24,50 litres/quintal.
Après avoir identifié les variables qualitatives qui ont un effet sur les rendements, nous avons étudié leurs effets sur l'ensemble des variables. L'analyse est menée à l'aide de l'analyse des correspondances multiples (ACM).

D'après les résultats de l'analyse des correspondances multiples (Fig. 1), nous remarquons la présence de deux groupes de facteurs ayant des effets significatifs : le premier regroupe les critères d'âge et l'origine de la variété, dont les rendements sont constatés comme plus élevés quand les variétés sont mixtes (de la région et hors région) et d'âge moyen. Le deuxième regroupe des critères liés à l'environnement, en particulier l'altitude et la pente, dont les rendements sont constatés comme plus élevés lorsque les exploitations sont 
situées à des niveaux d'altitude supérieurs à 600 mètres avec des pentes modérées.

\section{Conclusion}

Notre zone d'étude, la wilaya de Jijel, avec ses précipitations $1200 \mathrm{~mm} / \mathrm{an}$, avec son relief montagneux, renferme d'assez fortes potentialités pour le développement de l'oléiculture, particulièrement à des fins de production d'huile d'olive. La production des exploitations oléicoles de la région depuis le démarrage du programme de plantation montre leur faiblesse et leur irrégularité dans le temps. Pour identifier les principaux facteurs influençant les rendements oléicoles, nous avons réalisé une enquête sur terrain auprès des oléiculteurs de la région à l'aide d'un questionnaire, notre travail est basé sur un échantillon représentatif d'exploitations obtenu à partir d'une sélection raisonnée (une taille de référence pour une masse informationnelle importante; une bonne identification des pratiques culturales et des systèmes de cultures; accès facile à l'exploitation).

Les résultats des traitements statistiques montrent la présence de deux groupes de facteurs ayant des effets significatifs, le premier regroupe les critères d'âge et l'origine de la variété, dont les rendements sont constatés plus élevés quand les variétés sont sont mixtes (de la région et hors région) et d'âge moyen. Le deuxième regroupe des critères liés à l'environnement, en particulier l'altitude et la pente, dont les rendements sont constatés plus élevés lorsque les exploitations sont situées à des niveaux d'altitude supérieurs à 600 mètres avec des pentes modérées. Cette étude nous a permis d'identifier les principaux critères à prendre en considération pour augmenter les rendements et réaliser des gains économiques importants, notamment le choix de la variété et la régénération des oliveraies âgées et ainsi que les conditions environnementales particulièrement l'altitude et la pente.

\section{Références}

Barjol J-L. 2014. L'économie mondiale de l'huile d'olive. OCL 21(5): D502. Disponible sur https://www.ocl-journal.org/articles/ocl/ abs/2014/05/ocl140010/ocl140010.html.

Bouraine H. Analyse technico-économique de quelques exploitations oléicoles dans la commune El Adjiba, wilaya de Bouira. Mémoire d'ingénieur d'État en agronomie. Algérie: École Nationale Supérieure Agronomique (ENSA), 2012, p. 60.
Conseil oléicole international (COI). 2007. Techniques de production en oléiculture. Conseil oléicole international, $346 \mathrm{p}$. Disponible sur https://www.google.com/url? sa=t\&rct=j\&q=\&esrc= s\& source $=$ web \& $c d=1 \& v e d=2$ ahUKEwiB 1 PqZ - oPhAhX NyYUKHRDODTMQFjAAegQIAhAC\&url=http $\% 3 \mathrm{~A} \% 2 \mathrm{~F} \%$ 2Fwww.internationaloliveoil.org\%2Fstore \%2Fdownload $\%$ 2F42\&usg=AOvVaw3BL-bY36JHecvA12LzfcJU.

Direction des Services Agricoles (DSA). 2016. Statistiques agricoles de la wilaya de Jijel. Des données numériques fournies par le Bureau des statistiques agricoles. Algérie: Ministère de l'Agriculture, du Développement Rural et de la Pêche (MADRP).

Filippa MA. Formation et transformation des systèmes productifs locaux. Les spécificités des filières agroalimentaires. In: Communication au colloque: les systèmes agroalimentaires localisés : produits, entreprises et dynamiques locales. Gis-Syal, 2002, pp. 16-18.

Gharbi I, Issaoui M, Hammami M. 2014. La filière huile d'olive en Tunisie. OCL 21(2): D202. Disponible sur https://www.ocljournal.org/fr/?option=com_article\&url=/articles/ocl/full_html/ 2014/02/ocl130021/ocl130021.html\&Itemid=129\&lang=fr.

Hadjou L, Lamani O, Cheriet F. 2013. Labellisation des huiles d'olive algériennes : contraintes et opportunités du processus? New Medit 12(2): 35-45. Disponible sur http://wc3.iamb.it/share/img_new_ medit_articoli/941_35_hadjou.pdf.

Karray B, Abichou M. 2007. Fonctionnement, performances et devenir des exploitations oléicoles privées à Médenine (Tunisie). Rev Tropicultura 25(1): 26-30.

Karray B. 2012. Enjeux de lafilière oléicole en Tunisie et axes de développement dans le nouveau contexte politique. Les notes d'analyse du CIHEAM. $n^{\circ}$ 66-Avril 2012. Centre international de hautes études agronomiques méditerranéennes, p. 32. Disponible sur http://www.iamm.ciheam.org/ress_doc/opac_css/doc_num. php?explnum_id=9078.

Lamani O, Ilbert H. 2016. Spécificités de l'oléiculture en montagne (région kabyle en Algérie) : pratiques culturales et enjeux de la politique oléicole publique. In: Ater M, Essalouh L, Ilbert H, Moukhli A, Khadari B, eds. L'oléiculture au Maroc de la préhistoire à nos jours : pratiques, diversité, adaptation, usages, commerce et politiques. Montpellier: CIHEAM, 2016, pp. 149159 (Options Méditerranéennes : Série A. Séminaires Méditerranéens; n. 118).

Lazzeri Y. 2009. Les défis de la mondialisation pour l'oléiculture méditerranéenne. Conférence Centre Culturel Français de Tlemcen - Algérie (Novembre 2009). Institut Français à Tlemcen, p. 24

Mendil M. 2009. L'oléiculture: expériences algériennes, Filaha Innove 4: 6.

Meynard JM, Messéan A, Charlier A, et al. 2013. Freins et leviers à la diversification des cultures: étude au niveau des exploitations agricoles et des filières. OCL 20: D403. Disponible sur https:// www.ocl-journal.org/articles/ocl/abs/2013/04/oc120-4-D3/ ocl20-4-D3.html.

Citation de l'article : Lachibi M, Chehat F, Belhouadjeb FA. 2019. Les facteurs influençant le rendement oléicole : cas de la région de Jijel du Nord-Est algérien. OCL 26: 12. 